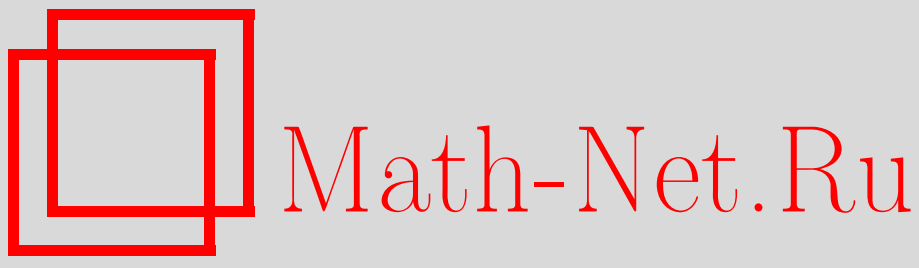

Р. С. Исмагилов, Примеры явного вычисления индуктивного предела семейства алгебр Ли, Матем. сб., 2000, том 191, номер 3, 53-64

DOI: https://doi.org/10.4213/sm463

Использование Общероссийского математического портала Math-Net.Ru подразумевает, что вы прочитали и согласны с пользовательским соглашением

http://www . mathnet.ru/rus/agreement

Параметры загрузки:

IP: 18.207 .199 .55

26 апреля 2023 г., 14:25:51 
УДК $515.16+517.9$

\author{
Р. С. Исмагилов
}

\title{
Примеры явного вычисления индуктивного предела семейства алгебр Ли
}

\begin{abstract}
В работе рассматриваются индуктивные пределы некоторых семейств алгебр Ли. Речь идет об алгебрах векторных полей на многообразии, сохраняющих форму объема либо симплектическую форму и сосредоточенных в координатных окрестностях. Изучается семейство всех коммутативных подалгебр алгебры Ли косоэрмитовых матриц порядка больше двух. Указывается явный вид индуктивных пределов.

Библиографояи: 5 названий.
\end{abstract}

\section{§1. Определения; описание результатов}

1.1. Индуктивные пределы. Это понятие общеизвестно в теории групп [1]. Для алгебр Ли определение совершенно аналогично; напомним его. Рассмотрим семейство алгебр Ли $L_{\alpha}$, и пусть для любых двух из них задан набор гомоморфизмов $\varphi_{\alpha \beta}: L_{\alpha} \rightarrow L_{\beta}$, причем набор может быть пустьм для некоторых $\alpha, \beta$. Представлением семейства $\left\{L_{\alpha}, \varphi_{\alpha \beta}\right\}$ в алгебре Ли $A$ назовем набор гомоморфизмов $f_{\alpha}: L_{\alpha} \rightarrow A$ такой, что $f_{\beta} \circ \varphi_{\alpha \beta}=f_{\alpha}$ и множество $\bigcup_{\alpha} f_{\alpha}\left(L_{\alpha}\right)$ порождает $A$ как алгебру Ли. Представление кратко записываем в виде $A,\left\{f_{\alpha}\right\}$. По определению индуктивный предел семейства $\left\{L_{\alpha}, \varphi_{\alpha \beta}\right\}$ - это представление $A^{\circ},\left\{f_{\alpha}^{\circ}\right\}$ со следуюшим свойством универсальности: для любого представления $A,\left\{f_{\alpha}\right\}$ сушествует такой гомоморфизм $p: A^{\circ} \rightarrow A$, что $f_{\alpha}=p \circ f_{\alpha}^{\circ}$ для всех $\alpha$.

Индуктивные пределы возникают, например, в следующей ситуации. Пусть $L$ - некоторая алгебра Ли, $\left\{L_{\alpha}\right\}$ - семейство ее подалгебр, замкнутое относительно пересечений; будем считать также, что множество $\bigcup L_{\alpha}$ порождает $L$ как алгебру Ли. Если $L_{\alpha} \subset L_{\beta}$, то определен гомоморфизм включения $i_{\alpha \beta}: L_{\alpha} \rightarrow L_{\beta}$. Требуется найти индуктивный предел семейства $\left\{L_{\alpha}\right\}$; здесь в обозначении семейства мы опускаем $i_{\alpha \beta}$.

1.2. Ограниченные индуктивные пределы. Добавим некоторые детали к ситуации, описанной в конце предыдушего пункта. Пусть $L$ - алгебра Ли, являющаяся локально выпуклым линейным топологическим пространством, $L_{\alpha}-$ семейство ее подалгебр, замкнутое относительно пересечений. Представление $A,\left\{f_{\alpha}\right\}$ этого семейства назовем ограниченным, если $A$ - локально выпуклое пространство и отображение $f: \bigcup L_{\alpha} \rightarrow A$, совпадаюшее с $f_{\alpha}$ на каждом $L_{\alpha}$, непрерьвно в нуле. Подчеркнем, что непрерьвность скобки Ли не требуется. Наконец, определим

Работа вьполнена при финансовой поддержке Российского фонда фундаментальных исследований (грант № 96-15-96249).

(C) Р.С. ИСМАГИЛОв 2000 
ограниченныц индуктивный предел семейства $\left\{L_{\alpha}\right\}$ как ограниченное представление, обладающее указанным выше свойством универсальности по отношению ко всем ограниченным представлениям.

Данное сейчас определение можно обобщить на случай произвольного семейства $\left\{L_{\alpha}, \varphi_{\alpha \beta}\right\}$ следующим образом.

Для каждого $\alpha$ зафиксируем множество $V_{\alpha} \subset L_{\alpha}$, порождающее $L_{\alpha}$ как линейное пространство. Представление $A,\left\{f_{\alpha}\right\}$ нашего семейства назовем ограниченным (относительно набора $V_{\alpha}$ ), если сушествует набор линейных функционалов $\left\{l_{\tau}\right\}$ на $A$ со следующими свойствами:

1) $\left\{l_{\tau}\right\}$ разделяет точки в $A$, т.е. для любого $a \neq 0$ существует такой $l_{\tau}$, что $l_{\tau}(a) \neq 0$

2) для любого $l_{\tau}$ сушествует такое число $M_{\tau}$, что $\left|l_{\tau}\left(f_{\alpha}(x)\right)\right|<M_{\tau}$ для всех $\alpha$ и для всех $x \in V_{\alpha}$.

Назовем ограниченным (по отношению $\kappa$ набору $V_{\alpha}$ ) индуктивным пределом нашего семейства ограниченное представление, обладаюшее указанным выше свойством универсальности по отношению ко всем ограниченным представлениям.

Мы будем прибегать к ограниченным индуктивным пределам в тех случаях, когда обычный индуктивньй предел приводит к аномально большим алгебрам Ли.

Переходим к примерам; они и составляют основное содержание этой работы.

1.3. Алгебры Ли векторных полей. В $\S 2$ рассматривается, во-первых, связное компактное многообразие $X$ с формой объема $v^{n}, n=\operatorname{dim} X$. Для любой области $Y \subset X$ через $\operatorname{Vect}\left(Y, v^{n}\right)$ обозначается алгебра Ли векторных полей, сохраняющих форму $v^{n}$ и сосредоточенных в $Y$. Возьмем семейство всех алгебр Ли вида $\operatorname{Vect}\left(Y, v^{n}\right)$, где $Y \simeq \mathbb{R}^{n}$. Если $Y_{1} \subset Y_{2}$, то имеем очевидное включение $\operatorname{Vect}\left(Y_{1}, v^{n}\right) \subset \operatorname{Vect}\left(Y_{2}, v^{n}\right)$. Требуется описать индуктивный предел этого семейства. Мы покажем, что решение поставленной задачи приводит к центральному расширению алгебры Ли $\operatorname{Vect}^{\nabla}\left(X, v^{n}\right)$ (она состоит из векторных полей, которые

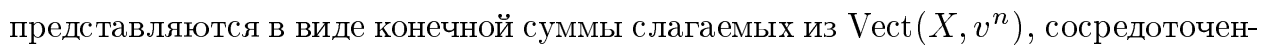
ных в координатных окрестностях $\left.Y \simeq \mathbb{R}^{n}\right)$ при помощи линейного пространства $H^{n-2}(X, \mathbb{R})$.

Заметим, что этот результат был сформулирован в приложении к работе автора [2], в которой рассматривались индуктивные пределы для групп диффеоморфизмов. Здесь мы приводим подробные доказательства.

Затем рассматривается симплектическое многообразие $\left(X, \omega^{2}\right)$ (связное и компактное), $\operatorname{dim} X=2 n$. Алгебры Ли $\operatorname{Vect}\left(Y, \omega^{2}\right), Y \simeq \mathbb{R}^{2 n}$, определяются аналогично предыдушим. Здесь, однако, удобнее заменить их изоморфными алгебрами $\mathscr{D}(Y)$ со скобкой Пуассона; $\mathscr{D}(Y)$ - это пространство бесконечно дифференцируемых функций, сосредоточенных в $Y$, а упомянутый изоморфизм устанавливается так: каждому векторному полю из $\operatorname{Vect}\left(Y, \omega^{2}\right)$ ставится в соответствие его гамильтониан с носителем в $Y$. Индуктивный предел полученного семейства алгебр Ли $\mathscr{D}(Y), Y \simeq \mathbb{R}^{2 n}$, есть алгебра Ли-Пуассона $\mathscr{D}(X)$. Более интересен индуктивньй предел семейства $\mathscr{D}^{\circ}(Y), Y \simeq \mathbb{R}^{2 n}$, где через $\mathscr{D}^{\circ}(Y)$ обозначено подпространство функций с нулевым интегралом относительно формы объема $\omega^{2 n}$ : индуктивным пределом оказьвается центральное расширение подалгебры Ли-Пуассона $\mathscr{D}^{\circ}(X)$ 
(функции с нулевьм интегралом относительно формы $\omega^{2 n}$ ) при помощи линейного пространства $H^{2 n-1}(X, \mathbb{R})$. Это известное расширение [3]; наш результат состоит в том, что оно возникает как индуктивный предел указанного семейства алгебр Ли.

1.4. Коммутативные подалгебры простой компактной алгебры Ли. Пусть $g$ - простая компактная алгебра Ли. Обозначим через Ab $g$ семейство всех ее коммутативных подалгебр. В каждой такой подалгебре возьмем шар радиуса 1 (в стандартной метрике алгебры $g$ ). Наша цель - найти ограниченный (по отношению к этим шарам) индуктивный предел семейства Ab g. Эта задача будет решена для алгебр Ли $\mathrm{su}(m), m>2$, и индуктивный предел приведет нас к алгебре Ли $T g[T]$, состоящей из полиномиальных токов $x_{1} T+x_{2} T^{2}+\cdots+x_{n} T^{n}, x_{k} \in g$. Наше доказательство не переносится на другие алгебры Ли; отметим, что оно существенно использует знаменитый результат Глисона о мерах на множестве подпространств гильбертова пространства (см. [4]).

\section{§2. Индуктивные пределы алгебр Ли векторных полей}

Всюду в этом параграфе через $X$ обозначается связное компактное бесконечно дифференцируемое многообразие, $\operatorname{dim} X<\infty$. Используются обычные обозначения: $\mathscr{D}(X)$ - пространство вешественных бесконечно дифференцируемых функций, $E^{k}(X)$ - пространство внешних $k$-форм, $d$-внешний дифференциал, $Z^{k}(X), B^{k}(X)$ - пространства замкнутых и точных $k$-форм, $H^{k}(X)=Z^{k}(X) / B^{k}(X), i_{\xi}$ - внутреннее умножение на векторное поле $\xi$. Для замкнутой $k$-формы $\alpha^{k}$ через $\left[\alpha^{k}\right]$ обозначается соответствующий элемент пространства $H^{k}(X, \mathbb{R})$. Если на $X$ дана форма объема $v^{n}$ либо симплектическая форма $\omega^{2}$, то через $\operatorname{Vect}\left(X, v^{n}\right)$ и соответственно $\operatorname{Vect}\left(X, \omega^{2}\right)$ обозначается алгебра Ли векторных полей, coxраняющих эту форму. Если $Y$ - область в $X, Y \simeq \mathbb{R}^{n}, n=\operatorname{dim} X$, то пространства функций, форм, векторных полей и т. д. с носителями в $Y$ обозначаются соответственно через $\mathscr{D}(Y), E^{k}(Y), \operatorname{Vect}\left(Y, v^{n}\right)$ и т. д. В случае многообразия с формой объема $\left(X, v^{n}\right)$ через $\operatorname{Vect}^{\nabla}\left(X, v^{n}\right)$ обозначим идеал в $\operatorname{Vect}\left(X, v^{n}\right), \operatorname{cocтоящий~из~вектор-~}$ ных полей, представимых в виде конечной суммы слагаемых, каждое из которых сосредоточено в некоторой области $Y \simeq \mathbb{R}^{n}$.

Мы будем пользоваться некоторыми соотношениями для отображений $d, i_{\xi}$ (см., например, [5; гл. 4]).

2.1. Векторные поля, сохраняющие форму объема. Пусть $v^{n}$ - форма объема на $X$. Рассмотрим линейное пространство $L(X)=E^{n-2}(X) / B^{n-2}(X)$.

Определим отображение $\gamma^{\circ}: E^{n-2}(X) \rightarrow \operatorname{Vect}^{\nabla}\left(X, v^{n}\right), \quad \gamma^{\circ}\left(\alpha^{n-2}\right)=\xi$, $d \alpha^{n-2}=i_{\xi} v^{n}$. Оно аннулирует пространство $B^{n-2}(X)$ и тем самым приводит к отображению

$$
\gamma: L(X) \rightarrow \operatorname{Vect}^{\nabla}\left(X, v^{n}\right) .
$$

Введем, далее, для любой области $Y \simeq \mathbb{R}^{n}$ отображение

$$
f_{Y}^{\circ}: \operatorname{Vect}\left(Y, v^{n}\right) \rightarrow L(X)
$$

поставив в соответствие векторному полю $\xi$ элемент $\alpha^{n-2}+B^{n-2}(X) \in L(X)$ такой, что $\gamma^{\circ}\left(\alpha^{n-2}\right)=\xi, \alpha^{n-2} \in E^{n-2}(Y)$. 
Теперь введем операцию $[\cdot, \cdot]$ на $L(X)$ по формуле

$$
\begin{gathered}
{\left[\alpha^{n-2}+B^{n-2}(X), \beta^{n-2}+B^{n-2}(X)\right]=i_{\xi} i_{\eta} v^{n}+B^{n-2}(X),} \\
\xi=\gamma^{\circ}\left(\alpha^{n-2}\right), \quad \eta=\gamma^{\circ}\left(\beta^{n-2}\right) .
\end{gathered}
$$

Применим равенство (3) к формам $\alpha^{n-2}, \beta^{n-2}$, сосредоточенным в области $Y \subset X, Y \simeq \mathbb{R}^{n}$. Учитывая равенство $d i_{\xi} i_{\eta} v^{n}=i_{[\xi, \eta]} v^{n},(3)$ можно переписать в виде $\left[f_{Y}^{0}(\xi), f_{Y}^{0}(\eta)\right]=f_{Y}^{0}([\xi, \eta])$. Отсюда следует, что операция, заданная в $L(X)$ формулой (3), есть скобка Ли и каждое из отображений (1), (2) есть гомоморфизм алгебр Ли.

Теорема 1. Алгебра Ли $L(X)$ вместе с вложениями (2) есть индуктивный предел семейства алгебр Ли $\left\{\operatorname{Vect}\left(Y, v^{n}\right), Y \simeq \mathbb{R}^{n}\right\}$.

ДокаЗАТЕльство. Возьмем представление $\left\{f_{Y}\right\}$ семейства $\left\{\operatorname{Vect}\left(Y, v^{n}\right)\right.$, $\left.Y \simeq \mathbb{R}^{n}\right\}$ в некоторой алгебре Ли $A$. Рассмотрим отображения

$$
p_{Y}^{\prime}=f_{Y} \circ \gamma^{\circ}: E^{n-2}(Y) \rightarrow A .
$$

Они согласованы в следуюшем смысле: если $Y_{1} \subset Y_{2}$, то отображения $p_{Y_{k}}^{\prime}, k=1,2$, действуют одинаковьм образомна $E^{n-2}\left(Y_{1}\right)$. Используя разложение единицы, без труда получаем такое линейное отображение $p^{\prime}: E^{n-2}(X) \rightarrow A$, что на каждом пространстве $E^{n-2}(Y), Y \simeq \mathbb{R}^{n}$, отображения $p_{Y}^{\prime}, p^{\prime}$ действуют одинаково. Поскольку отображение $p_{Y}^{\prime}$ аннулирует пространство $B^{n-2}(Y)$, то отображение $p^{\prime}$ аннулирует линейную оболочку таких пространств, т.е. пространство $B^{n-2}(X)$. В результате возникает отображение $p: L(X) \rightarrow A$. Это и есть гомоморфизм алгебр Ли такой, что $f_{Y}=p \circ f_{Y}^{\circ}$.

Теорема 1 доказана.

Исследуем подробнее алгебру Ли $L(X)$; это есть центральное расширение алгебры Ли $\operatorname{Vect}^{\nabla}\left(X, v^{n}\right)$ при помощи линейного пространства $H^{n-2}(X, \mathbb{R})$ (рассматриваемого как коммутативная алгебра Ли). Используя отображение $J^{n-1}: B^{n-2}(X) \rightarrow E^{n-1}(X)$, обратное справа к отображению $d$, мы получаем правое обратное для $\gamma$ по формуле $\xi \mapsto J^{n-1}\left(i_{\xi} v^{n}\right)+B^{n-2}(X)$. Оно позволяет найти 2-коцикл, отвечающий указанному расширению; он имеет вид

$$
\beta(\xi, \eta)=\left[i_{\xi} i_{\eta} v^{n}-J^{n-1}\left(i_{[\xi, \eta]} v^{n}\right)\right]
$$

Чтобы избавиться от неудобного (и неоднозначно определяемого) отображения $J^{n-1}$, возьмем произвольный элемент $\mu \in H^{2}(X, \mathbb{R})$ и представляющую его 2-форму $\theta^{2}$. Используя обычную двойственность между пространствами когомологий, образуем 2-коцикл $\langle\beta, \mu\rangle$. Выразив его в виде интеграла от произведения дифференциальных форм и отбросив слагаемое, которое содержит $[\xi, \eta]$ (это слагаемое есть тривиальный 2-коцикл на алгебре Ли $\left.\operatorname{Vect}^{\nabla}\left(X, v^{n}\right)\right)$, получаем следующий 2-коцикл с вешественными значениями:

$$
F_{\mu}(\xi, \eta)=\int_{X} i_{\xi} i_{\eta} v^{n} \wedge \theta^{2}, \quad \xi, \eta \in \operatorname{Vect}^{\nabla}\left(X, v^{n}\right)
$$


ПРЕДЛОЖЕНИЕ. Если $\mu \neq 0$, то 2-коцикл $F_{\mu}$ нетривиален (и, тем самымм, задает нетривиальное иентральное расширение алгебрь Ли $\operatorname{Vect}^{\nabla}\left(X, v^{n}\right)$ при помощи $\mathbb{R})$.

ДокАЗАТЕльство. Если 2-коцикл $F_{\mu}$ тривиален, то существует линейная функция $K$ на алгебре Ли $\operatorname{Vect}^{\nabla}\left(X, v^{n}\right)$ такая, что

$$
\int_{X} i_{\xi} i_{\eta} v^{n} \wedge \theta^{2}=K([\xi, \eta]), \quad \xi, \eta \in \operatorname{Vect}^{\nabla}\left(X, v^{n}\right)
$$

Возьмем область $Y \simeq \mathbb{R}^{n}$ в $X$. Тогда сушествует 1-форма $\lambda_{Y}^{1}$ на $Y$ такая, что $\theta^{2}=d \lambda_{Y}^{1}$. Для векторных полей, сосредоточенных в $Y$, равенство (4) принимает вид $\int_{X} i_{[\xi, \eta]} v^{n} \wedge \lambda_{Y}^{1}=K([\xi, \eta])$. Отсюда $K(\xi)=\int_{X} i_{\xi} v^{n} \wedge \lambda_{Y}^{1}, \xi \in \operatorname{Vect}\left(Y, v^{n}\right)$. Тем самым,

$$
K\left(\gamma^{\circ}\left(\alpha^{n-2}\right)\right)=\int_{X} d \alpha^{n-2} \wedge \lambda_{Y}^{1}=\int_{X} \alpha^{n-2} \wedge \theta^{2}, \quad \alpha^{n-2} \in E^{n-2}(Y) .
$$

Следовательно, $K\left(\gamma^{\circ}\left(\alpha^{n-2}\right)\right)=\int_{X} \alpha^{n-2} \wedge \theta^{2}$ для всех $\alpha^{n-2} \in E^{n-2}(X)$. В частности, для любой замкнутой формы $\alpha^{n-2}$ получаем равенство $\int_{X} \alpha^{n-2} \wedge \theta^{2}=0$. Но тогда 2-форма $\theta$ точна, а это противоречит тому, что $\mu \neq 0$. Предложение доказано.

2.2. Алгебра Ли векторных полей, сохраняющих симплектическую форму. Пусть $\left(X, \omega^{2}\right)$ - симплектическое многообразие (компактное и связное). Введем, как обычно, скобку Пуассона на $\mathscr{D}(X)$ по формуле $[u, v]=i_{\xi} d v$, где векторное поле $\xi$ определено равенством $i_{\xi} \omega^{2}=u$. Для наших целей удобна следующая запись скобки Пуассона:

$$
[u, v]=-n d u \wedge d v \wedge \frac{\omega^{2(n-1)}}{\omega^{2 n}}
$$

(поясним, что любая $2 n$-форма $\theta^{2 n}$ имеет вид $f \omega^{2 n}, f \in \mathscr{D}(X)$, и мы записываем функцию $f$ как $\left.\theta^{2 n} / \omega^{2 n}\right)$. Доказательство равенства (5) вытекает из очевидных соотношений $[u, v] \omega^{2 n}=i_{\xi} d v \wedge \omega^{2 n}=d v \wedge i_{\xi} \omega^{2 n}=n d v \wedge i_{\xi} \omega^{2} \wedge \omega^{2(n-1)}$. Скобка Пуассона приводит нас к алгебре Ли-Пуассона $\mathscr{D}(X)$, которая распадается в прямую сумму алгебр Ли $\mathbb{R} \oplus \mathscr{D}^{\circ}(X)$, где $\mathscr{D}^{\circ}(X)$ состоит из функций с нулевым средним относительно формы объема $\omega^{2 n}$.

Для любого открытого множества $Y \subset X$ обозначим через $\mathscr{D}^{\circ}(Y)$ подпространство функций из $\mathscr{D}(Y)$, удовлетворяющих условию $\int_{X} u \omega^{2 n}=0$. Тогда $\mathscr{D}(Y)$ преврашается в алгебру Ли, а $\mathscr{D}^{\circ}(Y)$ - в ее идеал. Наша цель - найти индуктивный предел семейства алгебр Ли $\left\{\mathscr{D}^{\circ}(Y), Y \simeq \mathbb{R}^{2 n}\right\}$.

С этой целью рассмотрим линейное пространство

$$
A(X)=E^{2 n-1}(X) / B^{2 n-1}(X)
$$


и превратим его в алгебру Ли. Предварительно введем некоторые отображения. Пусть

$$
\begin{gathered}
\gamma^{\circ}: E^{2 n-1}(X) \rightarrow \mathscr{D}^{\circ}(X), \\
\gamma^{\circ}\left(\alpha^{2 n-1}\right)=d \alpha^{2 n-1} / \omega^{2 n} .
\end{gathered}
$$

Это отображение аннулирует подпространство $B^{2 n-1}(X)$, что приводит к отображению

$$
\gamma: A(X) \rightarrow \mathscr{D}^{\circ}(X) .
$$

Далее, для любой области $Y \simeq \mathbb{R}^{2 n}$ определим отображение

$$
f_{Y}^{\circ}: \mathscr{D}^{\circ}(Y) \rightarrow A(X)
$$

по правилу

$$
f_{Y}^{\circ}(u)=\alpha^{2 n-1}+B^{2 n-1}(X), \quad \alpha^{2 n-1} \in E^{2 n-1}(Y), \quad \gamma^{\circ}\left(\alpha^{2 n-1}\right)=u .
$$

Введем теперь операцию $[\cdot, \cdot]$ в пространстве $A(X)$ по формуле

$$
\left[\alpha^{2 n-1}+B^{2 n-1}(X), \beta^{2 n-1}+B^{2 n-1}(X)\right]=-n u d v \wedge \omega^{2(n-1)}+B^{2 n-1}(X),
$$

где

$$
\gamma^{\circ}\left(\alpha^{2 n-1}\right)=u, \quad \gamma^{\circ}\left(\beta^{2 n-1}\right)=v
$$

Из $(5),(8)$ следует, что если $Y \simeq \mathbb{R}^{2 n}$, то $\left[f_{Y}^{\circ}(u), f_{Y}^{\circ}(v)\right]=f_{Y}^{\circ}([u, v])$. Отсюда вытекает, что операция (9) есть скобка Ли, а отображения (6), (7) - гомоморфизмы алгебр Ли.

Tеорема 2. Алгебра Ли $A(X),[\cdot, \cdot]$ вместе с гомоморфизмами (7) есть индуктивный предел семейства $\left\{\mathscr{D}^{\circ}(Y),[\cdot, \cdot], Y \simeq \mathbb{R}^{2 n}\right\}$.

Доказательство столь близко к доказательству теоремы 1 , что мы его опустим.

Рассмотрим подробнее алгебру Ли $A(X),[\cdot, \cdot]$. Она является центральным расширением алгебры Ли-Пуассона $\mathscr{D}^{\circ}(X)$ при помоши линейного пространства $H^{2 n-1}(X, \mathbb{R})$. Взяв линейный функционал на этом пространстве, мы получим центральное расширение алгебры Ли-Пуассона $\mathscr{D}^{\circ}(X)$ при помощи $\mathbb{R}$. Последнее расширение задается 2-коциклом $(\xi, \eta) \mapsto \int_{X} u \wedge d v \wedge \omega^{2(n-1)} \wedge \theta^{1}$, где $\theta^{1}$ - замкнутая 1-форма. Если эта форма не точна, то коцикл нетривиален (а потому указанное расширение нетривиально); доказательство этого утверждения аналогично доказательству предложения 1. 
§3. Коммутативные подалгебры алгебры Ли $\mathrm{su}(m), m>2$

Прежде чем перейти к основной теме, напомним знаменитый результат А. М. Глисона, существенно используемьй далее.

3.1. Теорема А. М. Глисона [4]. Пусть $H$ - гильбертово пространство (вещественное либо комплексное), $\operatorname{dim} H \geqslant 3$. Аддитивная мера на проекторах пространства $H$ - это комплекснозначная функция $P \mapsto f(P)(P$ - самосопряженньй проектор в $H)$ такая, что $f\left(P_{1}+P_{2}\right)=f\left(P_{1}\right)+f\left(P_{2}\right)$ при $P_{1} P_{2}=0$. Теорема А. М. Глисона утверждает, что если $f(P) \geqslant 0$ для всех $P$, mо $f(P)=\operatorname{Tr}(A P)$ для некоторого ядерного оператора $A$.

Отсюда вытекает, что если пространство $H$ конечномерно и аддитивная мера $f$ ограничена, то $f(P)=\operatorname{Tr}(A P)$ для некоторого линейного оператора $A$. Для доказательства этого утверждения достаточно применить теорему Глисона к аддитивным мерам $P \mapsto \operatorname{Re} f(P)+a \operatorname{dim} P(H), P \mapsto \operatorname{Im} f(P)+a \operatorname{dim} P(H)$, взяв достаточно большое число $a$.

3.2. Ограниченный индуктивный предел семейства $\mathrm{Ab} g, g=\mathrm{su}(m)$, $m>2$. Напомним, что $\mathrm{Ab} g$ - это семейство всех коммутативных подалгебр из $g=\mathrm{su}(m)$. В каждой алгебре из $\mathrm{Abg}$ возьмем шар $\|x\|<1$; здесь использована обычная норма на $g$. Опишем ограниченный (по отношению к семейству шаров) индуктивный предел этого семейства; подчеркнем, что $m>2$.

Обозначим через $T g[T]$ алгебру Ли, состоящую из всех многочленов $x_{1} T+x_{2} T^{2}+\cdots+x_{n} T^{n}, x_{k} \in g$. Скобка Ли определяется равенствами

$$
\left[x T^{m}, y T^{n}\right]=[x, y] T^{m+n}, \quad x, y \in g
$$

Для любой подалгебры $g^{\circ} \in \mathrm{Ab} g$ определим вложение

$$
f_{g^{\circ}}: g^{\circ} \rightarrow T g[T], \quad x \mapsto x T, \quad x \in g^{\circ}
$$

Теорема 3. Алгебра Ли Тg[T] вместе с гомоморфизмами (10) есть ограниченный индуктивный предел семейства $\mathrm{Ab} g, g=\mathrm{su}(m), m>2$.

Здесь пригодно любое из двух определений ограниченного индуктивного предела, данных в п. 1.2.

ДокаЗАтЕльство. Возьмем любое представление семейства $\mathrm{Ab} g$ в некоторой алгебре Ли $A$. Оно отождествляется с отображением $f: g \rightarrow A$ таким, что

$$
[f(x), f(y)]=0, \quad f(\alpha x+\beta y)=\alpha f(x)+\beta f(y) \text { при }[x, y]=0
$$

и, кроме того, для некоторого набора линейных функционалов $l_{\tau}$, определенных на $A$ и разделяющих точки из $A$, функция

$$
x \rightarrow l_{\tau}(f(x)), \quad x \in g,
$$

непрерывна в точке $x=0$. 
Лемма 1. Отображсение $f: g \rightarrow A$ линейно.

ДокАЗАТЕЛьство. Продолжим отображение $f$ на $u(m)$ (алгебру Ли всех косоэрмитовых матриц порядка $m)$ по формуле $f^{\prime}\left(i \alpha I_{m}+x\right)=f(x)$, где $I_{m}$ - единичная матрица, $\alpha \in \mathbb{R}, x \in g$. Ясно, что $f^{\prime}$ также удовлетворяет условию (11). Взяв один из упомянутых выше функционалов $l_{\tau}$, определим на множестве всех самосопряженных проекторов функцию $P \mapsto l_{\tau}\left(f^{\prime}(i P)\right)$. Из (11) следует, что это аддитивная мера на проекторах. Из непрерывности в нуле функции (12) вытекает ограниченность этой меры. Согласно теореме Глисона $l_{\tau}\left(f^{\prime}(x)\right)=\operatorname{Tr}(D x)$ для некоторого линейного оператора $D$, действующего в $g$. Итак, функция $x \mapsto l_{\tau}\left(f^{\prime}(x)\right)$, $x \in g$, линейна для некоторого набора линейных функционалов $l_{\tau}$, разделяющих точки в $A$. Тем самым отображение $f^{\prime}$ линейно. Лемма доказана.

Далее, перейдем от алгебр Ли $g=\mathrm{su}(m)$ и $A$ к их комплексификациям $g_{\mathbb{C}}=$ $\operatorname{sl}(m, \mathbb{C})$ и $A_{\mathbb{C}}$ и продолжим отображение $f: g \rightarrow A$ до $\mathbb{C}$-линейного отображения $f_{1}: g_{\mathbb{C}} \rightarrow A_{\mathbb{C}}$

Лемма 2. Существует $\mathbb{C}$-линейное отображсение $f_{2}: g_{\mathbb{C}} \rightarrow A_{\mathbb{C}}$ такое, что $\left[f_{1}(x), f_{1}(y)\right]=f_{2}([x, y])$ npu $x, y \in g_{\mathbb{C}}$.

ДоказАТЕЛьство. 1) Группа $G=\mathrm{SL}(m, \mathbb{C})$ и алгебра Ли $g_{\mathbb{C}}$ действуют на $g_{\mathbb{C}}$ (присоединенное представление). Отсюда возникает также действие на пространстве $g_{\mathbb{C}} \wedge g_{\mathbb{C}}$; действие элементов $g \in G$ и $a \in g_{\mathbb{C}}$ на вектор $x$ записываем в виде $\pi(g) x, \pi(a) x$. Легко подсчитать, что $g_{\mathbb{C}} \wedge g_{\mathbb{C}}$ разлагается в прямую сумму трех неприводимых подпространств $L_{k}, k=0,1,2$. Они задаются старшими (относительно верхнетреугольных матриц) векторами

$$
\begin{aligned}
& h_{0}=\sum_{i=2}^{m-1} e_{1 i} \wedge e_{i m}+\left(e_{11}-e_{m m}\right) \wedge e_{1 m} \\
& h_{1}=e_{1, m-1} \wedge e_{1 m}, \quad h_{2}=e_{1 m} \wedge e_{2 m} .
\end{aligned}
$$

(Здесь через $e_{i k}$ обозначены матричные единицы.)

2) Сушествует единственное линейное отображение $K: g_{\mathbb{C}} \wedge g_{\mathbb{C}} \rightarrow g_{\mathbb{C}}$ такое, что $K(x \wedge y)=[x, y]$ при $x, y \in g_{\mathbb{C}}$. Оно сплетает представления групшы $G=\mathrm{GL}(m, \mathbb{C})$ (и алгебры Ли $\left.g_{\mathbb{C}}\right)$, действующие в пространствах $g_{\mathbb{C}} \wedge g_{\mathbb{C}}$ и $g_{\mathbb{C}}$. Легко видеть, что

$$
K\left(h_{0}\right)=m e_{1 m}, \quad K\left(h_{1}\right)=0, \quad K\left(h_{2}\right)=0 .
$$

Отсюда вытекает, что отображение $K: L_{0} \rightarrow g_{\mathbb{C}}$ есть изоморфизм и $K\left(L_{1}\right)=\{0\}$, $K\left(L_{2}\right)=\{0\}$.

3 ) Сушествует единственное линейное отображение $F: g_{\mathbb{C}} \wedge g_{\mathbb{C}} \rightarrow g_{\mathbb{C}}$ такое, что $F(x \wedge y)=\left[f_{1}(x), f_{1}(y)\right]$ при $x, y \in g_{\mathbb{C}}$. Докажем, что $F\left(L_{1}\right)=\{0\}, F\left(L_{2}\right)=\{0\}$.

$\mathrm{C}$ этой целью любой четверке чисел $a, b, c, d \in\{1,2, \ldots, m\}, a \neq b, c \neq d$, поставим в соответствие элемент

$$
r_{a, b, c, d}=\left(e_{a a}-e_{b b}\right) \wedge\left(e_{c c}-e_{d d}\right)
$$


Через $S$ обозначим $G$-инвариантное подпространство, порожденное всеми $r_{a, b, c, d}$.

Подпространство $S$ есть линейная оболочка векторов

$$
\pi(u) r_{a, b, c, d}=u\left(e_{a a}-e_{b b}\right) u^{-1} \wedge u\left(e_{c c}-e_{d d}\right) u^{-1}, \quad u \in \mathrm{SU}(m) .
$$

Матрицы

$$
i u\left(e_{a a}-e_{b b}\right) u^{-1}, \quad i u\left(e_{c c}-e_{d d}\right) u^{-1}
$$

лежат в $\mathrm{su}(m)$ и перестановочны. Отсюда следует (по определению отображения $F$ ), что

$$
F\left(\pi(u) r_{a, b, c, d}\right)=0
$$

Значит, $F(S)=\{0\}$.

Докажем, что $L_{1} \subset S, L_{2} \subset S$. Без труда проверяются равенства

$$
\pi\left(e_{1 m}\right) \pi\left(e_{1, m-1}\right) r_{1, m, m-1, m}=3 h_{1}, \quad \pi\left(e_{2 m}\right) \pi\left(e_{1 m}\right) r_{1,2,2, m}=3 h_{2}
$$

напомним, что векторы $h_{1}, h_{2}$ были введены выше. Тем самым, $h_{1} \in S, h_{2} \in S$ и, следовательно, $L_{1} \subset S, L_{2} \subset S$. Но тогда $F\left(L_{1}\right)=\{0\}, F\left(L_{2}\right)=\{0\}$, что и утверждалось.

4) Мы доказали, что ker $K=L_{1} \oplus L_{2}, \operatorname{ker} F \supset L_{1} \oplus L_{2}$; таким образом, $\operatorname{ker} K \subset$ $\operatorname{ker} F$. Но тогда $F=f_{2} \circ K$ для некоторого линейного отображения $f_{2}: g_{\mathbb{C}} \rightarrow A$. Отсюда $\left[f_{1}(x), f_{1}(y)\right]=f_{2}([x, y])$ при $x, y \in g_{\mathbb{C}}$, чем доказана лемма 2.

ЛЕмма 3. Пусть для некоторого $n \geqslant 2$ дань $\mathbb{C}$-линейные отображсения

$$
f_{i}: g_{\mathbb{C}} \rightarrow A_{\mathbb{C}}, \quad 1 \leqslant i \leqslant n,
$$

удовлетворяющие условиям

$$
\left[f_{i}(x), f_{k}(y)\right]=f_{i+k}([x, y])
$$

для всех $i, k \in\{1,2, \ldots, n-1\}$ таких, что $i+k \leqslant n$. Тогда существует такое $\mathbb{C}$-линейное отображсение $f_{n+1}: g_{\mathbb{C}} \rightarrow A_{\mathbb{C}}$, что

$$
\left[f_{i}(x), f_{k}(y)\right]=f_{n+1}([x, y])
$$

nрu $i, k \in\{1,2, \ldots, n\}, i+k=n+1$.

ДокАЗАтЕЛьство. В дальнейшем через $H$ обозначается подалгебра диагональных матриц из $g_{\mathbb{C}} ;$ положим

$$
h_{a b}=e_{a a}-e_{b b}, \quad 1 \leqslant a \neq b \leqslant n .
$$

Используется стандартная билинейная форма Киллинга $(x, y)$ на алгебре Ли $g_{\mathbb{C}}=$ $\operatorname{gl}(m, \mathbb{C})$. 
Из (13) вытекает равенство

$$
\left[f_{i}(h), f_{k}\left(e_{a b}\right)\right]=\left(h, h_{a b}\right) f_{i+k}\left(e_{a b}\right), \quad i+k \leqslant n .
$$

Умножая его слева (разумеется, под умножением понимается применение скобки Ли) на $f_{p}\left(e_{c d}\right)$ и на $f_{p}\left(h^{\prime}\right), h^{\prime} \in H, c \neq d, i+k+p=n+1$, приходим к равенствам

$$
\begin{gathered}
\left(h, h_{c d}\right)\left[f_{k}\left(e_{a b}\right), f_{i+p}\left(e_{c d}\right)\right]+\left[f_{i}(h), f_{p+k}\left(\left[e_{c d}, e_{a b}\right]\right)\right] \\
=\left(h, h_{a b}\right)\left[f_{p}\left(e_{c d}\right), f_{i+k}\left(e_{a b}\right)\right], \\
\left(h^{\prime}, h_{a b}\right)\left[f_{i}(h), f_{p+k}\left(e_{a b}\right)\right]=\left(h, h_{a b}\right)\left[f_{p}\left(h^{\prime}\right), f_{i+k}\left(e_{a b}\right)\right], \\
i+k+p=n+1 .
\end{gathered}
$$

Дальнейшие рассуждения (они основаны на равенствах (14)-(16)) разобьем на шаги.

Шаг 1. Положим $D_{i}(a, b)=\left[f_{i}\left(e_{a b}\right), f_{n+1-i}\left(e_{b a}\right)\right], 1 \leqslant i \leqslant n, 1 \leqslant a \neq b \leqslant n$. Докажем, что

$$
\begin{gathered}
D_{i}(a, b)=D_{1}(a, b), \quad 1 \leqslant i \leqslant n \\
D_{1}(a, b)+D_{1}(b, a)=0, \\
D_{1}(a, b)+D_{1}(b, c)+D_{1}(c, a)=0, \quad a \neq b, \quad a \neq c, \quad b \neq c .
\end{gathered}
$$

Для этого применим равенство $(15)$, полагая $(c, d)=(b, a), h=h_{a b}$. Получим (после очевидных преобразований) $D_{i+k}(a, b)-D_{k}(a, b)=\frac{1}{2}\left[f_{i}\left(h_{a b}\right), f_{n+1-i}\left(h_{a b}\right)\right]$, $i+k \leqslant n, a \neq b$. Зафиксируем числа $a, b$ и обозначим $D_{i}(a, b)$ через $D_{i}$, а правую часть этого равенства - через $r_{i}$. Тогда $D_{i+k}-D_{i}=r_{i}, r_{i}+r_{n+1-i}=0$; здесь $i, k \in\{1,2, \ldots, n-1\}, i+k \leqslant n$. Первое из этих равенств дает $r_{i}=i r_{1}$, а второе приводит к равенству $r_{1}=0$. Отсюда $r_{i}=0, D_{i+k}=0$, чем доказано утверждение (17).

Равенство (18) следует из соотношений

$$
D_{1}(a, b)=\left[f_{1}\left(e_{a b}\right), f_{n}\left(e_{b a}\right)\right]=-D_{n}(b, a)=-D_{1}(b, a) .
$$

Для доказательства (19) используем равенство $\left[f_{1}\left(e_{a b}\right), f_{1}\left(e_{b c}\right)\right]=f_{2}\left(e_{a c}\right)$, вытекаюшее из (13). Умножим обе части на $f_{n-1}\left(e_{c a}\right)$, причем в левой части применим тождество Якоби и определение элементов вида $D_{i}(a, b)$; используя, далее, свойство (17), приходим к равенству (19).

Шаг 2. Докажем, что

$$
\left[f_{i}(h), f_{n+1-i}\left(h^{\prime}\right)\right]=0 \text { при } 1 \leqslant i \leqslant n \quad \text { и } h, h^{\prime} \in H .
$$

Для этого в равенстве $(15)$ положим $(c, d)=(b, a)$. Это приводит к равенству $\left[f_{i}(h), f_{n+1-i}\left(h_{a b}\right)\right]=0$ при $1 \leqslant i \leqslant n, a \neq b$. Так как элементы $h_{a b}$ порождают линейное пространство $H$, то это приводит к (20). 
Шаг 3. После всех предварительных рассуждений определим требуемое леммой отображение $f_{n+1}$. Сначала зададим его на элементах $h_{a b}, a \neq b$, по формуле $f_{n+1}\left(h_{a b}\right)=D_{1}(a, b)$. Из соотношений $(17)-(19)$ вытекает, что этим определяется линейное отображение $f_{n+1}: H \rightarrow A_{\mathbb{C}}$. Далее, из равенства (16) следует, что отношение

$$
\frac{\left[f_{i}(h), f_{n+1-i}\left(e_{a b}\right)\right]}{\left(h, h_{a b}\right)}
$$

(разумеется, мы предполагаем, что знаменатель отличен от нуля) не зависит от $h, i$. Обозначим это отношение через $f_{n+1}\left(e_{a b}\right)$. В результате мы получаем линейное отображение $f_{n+1}: g_{\mathbb{C}} \rightarrow A_{\mathbb{C}}$.

Покажем, что построенное отображение $f_{n+1}$ удовлетворяет условию (13a). Из предыдущего построения вытекает, что (13a) выполняется при $x \in H$. Осталось проверить это условие для случая $x=e_{a b}, y=e_{c d}, a \neq b, c \neq d$. Пусть сначала $a \neq d, b \neq c$. В равенстве (15) положим $h=h_{a b}$. Получим $\left[f_{p}\left(e_{c d}\right), f_{n+1-p}\left(e_{a b}\right)\right]=0$, что и требуется. Если $b \neq c, a=d$, то в $(15)$ положим $\left(h, h_{c a}\right)=0,\left(h, h_{a b}\right)=1$. Это приводит к доказьваемому равенству

$$
\left[f_{p}\left(e_{c a}\right), f_{i+k}\left(e_{a b}\right)\right]=f_{n+1}\left(e_{c b}\right) .
$$

Наконец, в случае $a=d, b=c$ нужное равенство вытекает из определения элемента $f_{n+1}\left(h_{a b}\right)$.

Леммы 2,3 позволяют построить бесконечную последовательность линейных отображений $f_{k}: g \rightarrow A, k \geqslant 1$, таких, что

$$
\left[f_{i}(x), f_{k}(y)\right]=f_{i+k}([x, y]), \quad i, k \in\{1,2, \ldots\}, \quad x, y \in g_{\mathbb{C}}
$$

Заметим, что все отображения $f_{i}$ вещественны, т.е. отображают вещественную подалгебру $g \subset g_{\mathbb{C}}$ в вешественную подалгебру $A \subset A_{\mathbb{C}} ;$ для отображений $f_{k}$ это вытекает из соотношений (21), а для отображения $f_{1}$ - очевидно.

Теперь завершим доказательство теоремы 3. Зададим линейное отображение $p: T g[T] \rightarrow A$ формулой $p\left(x T^{k}\right)=f_{k}(x), x \in g, k \geqslant 1$. Из (21) вытекает, что $p-$ гомоморфизм алгебр Ли. Имеем равенство $p(x T)=f(x), x \in g$, где $f: g \rightarrow A-$ отображение, с которого мы начали доказательство теоремы. Теорема доказана.

\section{Заключение (об образующих и соотношениях в алгебре Ли)}

Индуктивные пределы дают (иногда) способ записи алгебры Ли при помощи образующих и соотношений. Поясним это на примере алгебры Ли $\operatorname{Vect}^{\nabla}\left(X, v^{n}\right)$, используя теорему 1. Эта алгебра порождается парами $(Y, \xi)$, где $Y \simeq \mathbb{R}^{n}, \xi \in$ $\operatorname{Vect}\left(Y, v^{n}\right)$. Образующие подчинены очевидным соотношениям

$$
[(Y, \xi),(Y, \eta)]=(Y,[\xi, \eta]), \quad\left(Y_{1}, \xi\right)=\left(Y_{2}, \xi\right) \text { при } Y_{1} \subset Y_{2} .
$$

Однако согласно теореме 1 эти соотношения (их можно назвать “локальными”) приводят не к алгебре $\operatorname{Vect}^{\nabla}\left(X, v^{n}\right)$, а к ее центральному расширению $L(X)$. Чтобы получить алгебру Ли $\operatorname{Vect}^{\nabla}\left(X, v^{n}\right)$, следует добавить еще "глобальные" соотношения - с целью избавиться от центра $H^{n-2}(X)$; их количество равно величине $\operatorname{dim} H^{n-2}(X)$. 


\section{Список литературы}

1. Серр Ж.-П. Деревья, амальгамы и $\mathrm{SL}_{2}$ // Математика. 1974. Т. 18. № 1. С. 3-51.

2. Исмагилов P. С. О группах диффеоморфизмов, сохраняющих объем // Изв. АН СССР. Сер. матем. 1980. Т. 4. С. $831-867$.

3. Kirillov A. A. La géometrie des moments pour les groupes de difféomorphismes. Operator algebras, unitary representations, enveloping algebras, and invariant theory. Proc. Colloq. in Honour of J. Dixmier, Paris/Fr. 1989 // Prog. Math. 1990. V. 92. P. 73-83.

4. Gleason A. M. Measures on the closed subspaces of a Hilbert space // J. Math. Mech. 1957. V. 6. №6. P. $885-895$.

5. Годбийон K. Дифференциальная геометрия и аналитическая механика. М.: Мир, 1973.

Московский государственньй

технический университет им. Н.Э. Баумана
Поступила в редакцию 24.02 .1999 